\title{
Multipactor breakdown in microwave pulses
}

\author{
J. Rasch ${ }^{1}$, D. Anderson ${ }^{1}$, and V. E. Semenov ${ }^{2}$ \\ 1) Chalmers University of Technology, Göteborg, Sweden \\ 2) Institute of Applied Physics, Nizhny Novgorod, Russia
}

A theoretical investigation is made of the effect of finite pulse length on the multipactor breakdown condition for microwave signals. The analysis considers the case of multipactor between two parallel metallic plates, using the classical resonance theory. It is found that decreasing pulse length tends to successively eliminate higher order resonance zones and lead to a "pulse hardening" of lower order modes where the multipactor-prone resonance zones shrink and the lower boundary breakdown field of the zones smoothly increases as the pulse becomes shorter, eventually to completely eliminate the multipactor effect for sufficiently short pulses.

\section{Introduction}

Multipactor discharges in microwave components constitute a severe breakdown problem in many modern microwave systems involving high powers, a typical example being space borne RF equipment for communication purposes. The discharge is caused by free electrons oscillating in vacuum between surfaces in the device, knocking out secondary new electrons when hitting the surfaces, and creating an avalanche-like growth of the electron density in the device, provided certain threshold conditions are fulfilled. The concomitant breakdown discharge tends to generate noise, change the device impedance, heat the device walls and may even permanently damage the hardware in the devices. Thus, an important part of the design and development of RF components is to establish the critical RF power at which the breakdown process is initiated. This step has become increasingly important in view of the development of modern space borne microwave technologies towards higher and higher data rates which makes increasing RF power levels necessary in order to maintain a sufficient carrierto-noise ratio.

Most investigations of the multipactor phenomenon have been made for continuous $(\mathrm{CW})$ operation, i.e. the microwave power is constant in time. In this situation it is clear that for any value of the secondary emission coefficient above its threshold value, the electron density will eventually grow to very high values. In fact, the successive electron growth can be described by the expression

$$
\frac{N(m)}{N_{0}}=\sigma^{m}=\exp (m \ln \sigma)
$$


where $N(m)$ is the number of electrons after $m$ impacts, $N_{0}$ the initial number of electrons and $\sigma$ the coefficient of the electron secondary emission yield (SEY) averaged over a bunch of impacting resonant electrons. The impact number $m$ is a discrete, but "time like", variable since it is directly related to the time needed to make $m$ crossings between the plates. Obviously, a necessary and sufficient condition for electron density growth is $\sigma>1$, where the electron impact velocity at the surfaces determines the relevant value of $\sigma$.

However, in many microwave applications, the microwave power is not constant in time as e.g. in pulsed operation involving repetitive pulses of finite length in time separated by time slots of zero power. In such situations, breakdown can only occur provided the electron avalanche has time to grow to a large enough value within the duration of the pulse, or if the time between pulses is short enough for the electron population not to decay completely between pulses. The latter case can from a multipactor point of view, depending on the situation, be more dangerous than the single pulse case. An investigation of this case is presented in [1], where it was shown that this effect is very sensitive to the reflection of low energy electrons between pulses. We will only be concerned with the first case, basically assuming that the time between pulses is long enough to eliminate this electron build-up effect. This case is simpler than the one in [1], for it eliminates the need for making assumptions about the dynamics of low energy electrons, in particular the reflection of electrons.

When designing high power microwave systems, the so called "20 gap crossings rule" is used as an engineering "rule of thumb" for assessing the risk of multipactor breakdown in pulsed microwave signals. This rule states that [2] "As long as the duration of the high power peak and the mode order of the gap are such that no more than twenty gap-crossings can occur during the high power peak, then multipaction-generated noise should remain well below thermal noise (in a $30 \mathrm{MHz}$ band)." This rule is used widely within the space community [3] and has its origin in numerical simulations performed between parallel plates for two different values of the maximum SEY: 1.66 and 2.22 [2]. The physical mechanism behind the generation of noise is the build-up of free electrons. So by using our knowledge of the number of gap crossings and the SEY we can estimate how much the electron population must grow for multipactor effects to appear. Given an average SEY of about 2 the multiplication factor (or exponentiation) factor $\exp (m \ln \sigma)$ becomes of the order of $10^{6}$. The number $10^{6}$ is in no way meant as an exact estimate. In every microwave system there will be a critical electron number density, and by relating this to the ambient electron density one could in principle find this number. But it will be very system specific, as it will depend on the ambient electron density, the system geometry, the detection method etc. However, the quantitative results are fairly unsensitive to this factor, and the qualitative results are not sensitive at all. Throughout this paper we will use the value $10^{6}$, but the formulas are easily adapted to any number, and in the last section we will compare results of using $10^{4}, 10^{6}$ and $10^{8}$.

Microwave signals with even more complicated time variation than that of repeated pulses arise in several other technically important situations e.g. it may 
result from the interference of many signals in multi-carrier operation scenarios or simply due to the coding of information in signals in microwave communication systems. The corresponding time evolution of the electron avalanche may be very complicated and it becomes difficult to establish a simple threshold condition for microwave breakdown. It is intuitively clear that a very dangerous case is when many signals in a multi-carrier system add constructively to give rise to high power peaks, which however tend to be of limited time duration. The 20 gap-crossing rule is a first effort to establish a breakdown criterion for this type of situation. Although physically pregnant, it is clear that this rule can only be regarded as a tentative recommendation and not as a well established criterion based on detailed investigations of the breakdown problem. A few studies have been published where more complicated signals (multicarrier) are considered both numerically and experimentally in several geometries [4], $[5],[6]$ and [7]. In essence, such signals will produce intervals where the field is above the CW breakdown threshold, and intervals where it is below. This can affect the electrons in basically the same way as a pure pulsed signal; which was the reasoning behind the simplified model used in [1]. A further complication is introduced by the use of phase shift keying of the different carriers. Depending on the amplitude, frequency and phase shift rate of the different carriers, any number of complex situations will arise, which may significantly change the classical resonance conditions. So far very little research has been performed on multipactor in signals with phase shift keying, even for the most simple case of one carrier [8].

So far, nobody has considered what the fundamental implications will be by applying a pulsed signal to the classical resonant parallel plate case. The present analysis studies in more detail the requirements for multipactor breakdown in microwave pulses of finite length in the simple case of parallel metallic plates within the resonant regime. This system, and the results concerning multipactor, has the benefit of being well known and thoroughly investigated, which means that previous results can be used without performing any complicated derivations. It is shown that similarly to the case of microwave breakdown in gases (corona breakdown) [9], a shortening of the pulse length leads to a "pulse hardening" effect in the sense that the electric field needed to cause breakdown increases as the pulse length becomes shorter. However, since in the multipactor case the SEY curve has a non-monotonic dependence on electron impact energy and the electron oscillations between the plates are required to be resonant, the resulting breakdown curve exhibits several new features as compared to the corona case e.g. a step-like behaviour when the breakdown process changes from one resonance order to another.

\section{Breakdown in microwave pulses}

In a resonant multipactor discharge between two parallel plates, one or several thin sheaths of electrons are moving between the two surfaces. In a resonant multipactor of order $n(n=1,3,5 \ldots)$, the electron sheath crosses the gap 
(length $d$ ) in a time $\tau_{n}=n \pi / \omega$, where $\omega$ is the field angular frequency. Upon each impact, the number of electrons in the sheath, $N$, will change to $\sigma_{n} N$, where $\sigma_{n}$ is the average SEY corresponding to the particular impact velocity of the sheath. The number of electrons after $m$ gap crossings will thus be

$$
N_{m}=N_{0}\left(\sigma_{n}\right)^{m}
$$

Under CW conditions, the necessary criterion for an avalanche to develop is simply $\sigma_{n}>1$. However, in a pulsed signal, this criterion is not sufficient. As mentioned above, in practice it is often assumed that multipactor effects will become important and detectable when $N_{m} / N_{0} \approx 10^{6}$. This means that given a certain $\sigma_{n}$, the electron sheath must complete

$$
m=\frac{\ln N_{m} / N_{0}}{\ln \sigma_{n}} \approx \frac{14}{\ln \sigma_{n}}
$$

crossings before the discharge becomes noticeable. Conversely, for a given number of gap crossings, $m$, there is a lower value for $\sigma_{n}$, under which multipactor will not be detectable. This value is

$$
\sigma_{n, \text { low }}=\left(\frac{N_{m}}{N_{0}}\right)^{1 / m} \approx 10^{6 / m}
$$

Thus, multipactor discharges are only possible when $\sigma_{n}>\sigma_{n \text {,low }}$. Clearly, when $m \rightarrow \infty$, the CW value $\sigma_{n, \text { low }}=1$ is regained.

In a resonant discharge, where the electrons cross the gap in the time $\tau_{n}=$ $n \pi / \omega$, the electron sheath will cross the gap $m=\left[\tau_{p} / \tau_{n}\right] \approx \tau_{p} / \tau_{n}$ times during the pulse time $\tau_{p}$, where $\left[\tau_{p} / \tau_{n}\right]$ denotes the integer part of $\tau_{p} / \tau_{n}$. For most wall materials the maximum SEY, $\sigma_{\max }$ is $\sigma_{\max } \sim 5$, which means that $m>$ $\min \left(\tau_{p} / \tau_{n}\right) \approx 10$ and the error introduced by the approximation $\left[\tau_{p} / \tau_{n}\right] \approx \tau_{p} / \tau_{n}$ is small. The criterion for multipactor breakdown in a pulse of finite length can thus be given as

$$
\sigma_{n}>\sigma_{n, \text { low }} \approx 10^{6 \tau_{n} / \tau_{p}}
$$

Clearly, when $\sigma_{n \text {,low }}>\sigma_{\max }$ is satisfied for all $n$, multipactor breakdown is totally suppressed.

Application of the breakdown condition given by Eq. (5) leads to modified criteria for multipactor breakdown and to Hatch-Williams diagrams [10] where the size of the resonant zones as well as the number of resonant zones depend on the pulse length. Section 3 will present a summary of the classical theory concerning the resonant multipactor charts, and it will also be shown how these charts change for finite pulse lengths. In particular, the breakdown power as a function of pulse length is found to exhibit characteristic jumps between different resonance levels, where the exact form of the resulting curve will depend strongly on the gap distance and the field frequency. This result is analyzed in more detail in section 5 . 


\section{Resonance theory and multipactor zones}

The classical resonance theory for multipactor rests on the assumption that the emission velocity of the secondary electrons is fixed to some value $v_{e}$, and that the spread in this quantity is of little importance. This means that the theory can be applied with good accuracy for the first resonance zones, provided $\Delta v_{e} d \ll\left(v_{e}+v_{\omega}\right)^{2} \omega /(2 \pi)[11]$, where $\Delta v_{e}$ is the average spread in the emission velocity $v_{e}$ and $v_{\omega}$ is the electron oscillatory velocity in the microwave field. When this criterion is fulfilled, the resonant zones can be found by considering three different effects. First of all, electrons participating in a classical resonant discharge must cross the gap in an odd number of field half cycles. The electron trajectories are determined by the solution of the equation of motion for the electrons and given by [11]

$$
z(t)=\frac{v_{\omega}}{\omega}\left(\sin \omega t-\sin \omega t_{e}\right)+\left(v_{e}-v_{\omega} \cos \omega t_{e}\right)\left(t-t_{e}\right)
$$

It should be emphasized that due to the phase stability of the electron motion, there are certain phase regions where a small perturbation of the electron emission phase results in a smaller perturbation in the impact phase and subsequent stable resonant motion. Classically, the stability factor, $G$,

$$
G \equiv\left|\frac{v_{e}-v_{\omega} n \pi \sin \omega t_{e}}{v_{e}-2 v_{\omega} \cos \omega t_{e}}\right|
$$

is required to be smaller than unity. However, a larger value for $G$ can be allowed in the presence of a larger SEY [12]. Thus, the condition

$$
\sigma_{n}>G
$$

can be taken as the appropriate criterion for phase stability.

The final requirement for multipactor breakdown to be possible in a pulsed signal is that the SEY should be larger than the critical value derived in the previous section, i.e.

$$
\sigma_{n}>\sigma_{n, \text { low }} \approx 10^{6 \tau_{n} / \tau_{p}}
$$

Actually it is more convenient to use $\tau_{n}=n \pi / \omega=n T / 2$, where $T$ is the signal period, in which case the condition reads

$$
\sigma_{n}>\sigma_{n, \text { low }} \approx 10^{3 n T / \tau_{p}}
$$

Figs. 1 - 4 show the dependence of the size of the classical resonance zones on the ratio between the pulse length and the field period. In these figures we also include a common approximation for the lower envelope of the resonance zones [13]. This approximation is simply the equality between the maximum impact velocity and the first cross over velocity, $v_{1}$, i.e.

$$
2 v_{\omega}+v_{e}=v_{1}
$$


Fig. 1 shows the resonant zones for $\mathrm{CW}$ conditions, the so-called HatchWilliams diagram. In practice, this depiction is not quite true and only the first couple of resonance zones have been verified experimentally (see e.g. [10]). In addition there will be hybrid zones between the classical resonance zones [12]. When using the resonant theory under CW conditions it is not necessary to have a detailed knowledge of the SEY curve for the material in question, whereas in the pulsed case, the shape of the curve and the maximum SEY becomes of great importance. Throughout this paper we will use the simple Vaughan approximation [14] for the SEY as a function of the electron impact velocity

$$
\sigma=\sigma_{\max }[\epsilon \exp (1-\epsilon)]^{\beta}
$$

where $\epsilon=W_{i} / W_{\max }, W_{i}$ is the electron impact energy, $W_{\max }$ is the electron impact energy corresponding to the secondary yield maximum, and $\beta=0.62$ if $W_{i} \leq W_{\max }$, and 0.25 if $W_{i}>W_{\max }$. This simple SEY curve is thus characterized completely by $\sigma_{\max }$ and $W_{i}$, or alternatively (and more frequenctly used) by $\sigma_{\max }$ and $W_{1}$, where $W_{1}$ is the electron impact energy where the SEY is unity, the so-called first cross over energy.

The SEY depends on the impact velocity of the electrons, above the first cross over velocity, $v_{1}$, the SEY is above unity, as the impact velocity increases the SEY curve goes through a maximum, and eventually, when the velocity becomes higher than the second cross over velocity, $v_{2}$, the SEY drops below unity again. It is standard practice to use the cross over energy instead of velocity. In this case we have $W_{1}=m v_{1}^{2} / 2$, and $W_{2}=m v_{2}^{2} / 2$, where $m$ is the electron mass. The same goes for the average emission energy of the secondary electrons, $W_{e}=m v_{e}^{2} / 2$.

In Figs. 2-4 one can see the appearance of the Hatch-Williams diagrams for pulse lengths between 40 and 7 field periods. Fig. 2 shows the diagram for a pulse length of 40 field periods. Even such a long pulse tends to eliminate all resonance zones with $n>5$, simply because those trajectories need too many field periods to cross the gap and never reach the critical number regardless of the power. In addition to the disappearance of the higher order zones, the resonant zones that still exhibit multipactor breakdown have shrunk. The upper boundaries have been shifted down in voltage, and the lower ones up. In Fig. 3 the pulse length is 20 field periods, and only the zones corresponding to $n=1$ and $n=3$ remain. Finally, in Fig. 4, the pulse length is 7 field periods. Only the first zone remains and it has shrunk significantly. In this case the electrons are only able to cross the gap 14 times during the pulse, which requires a rather high SEY for the electron population to reach the assigned critical value. Given the physical parameters, multipactor becomes impossible when $\tau_{p} / T=6.3$. However, as the number of electron transits is a discrete quantity, multipactor would be impossible for $\tau_{p} / T<7$. 


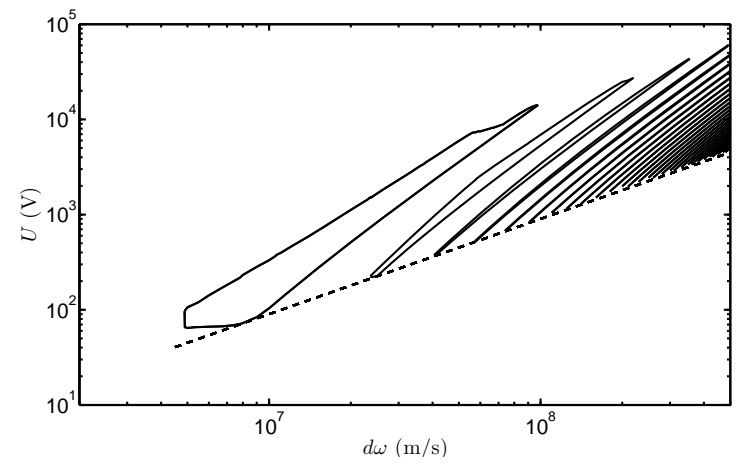

Figure 1: The appearance of the classical Hatch-Williams diagram for parallel plates under $\mathrm{CW}$ conditions, where the spread in emission velocity is assumed to be zero. The physical parameters are $W_{1}=50 \mathrm{eV}, W_{e}=3 \mathrm{eV}$, and $\sigma_{\max }=3$. The dashed straight line represents the breakdown voltage predicted by Eq. (11)

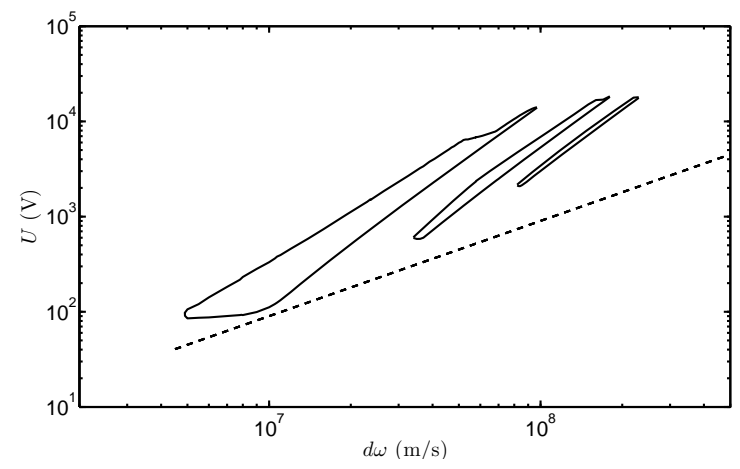

Figure 2: The appearance of the classical Hatch-Williams diagram for a pulse length of 40 field periods. The physical parameters are the same as in Fig. 1.

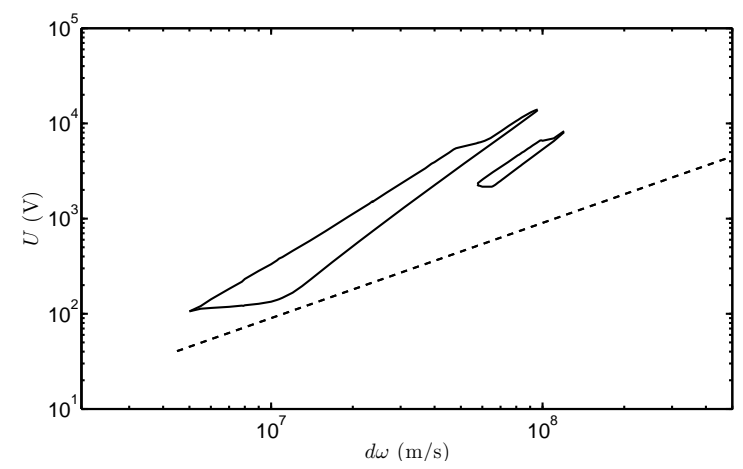

Figure 3: The appearance of the classical Hatch-Williams diagram for a pulse length of 20 field periods. The physical parameters are the same as in Fig. 1. 


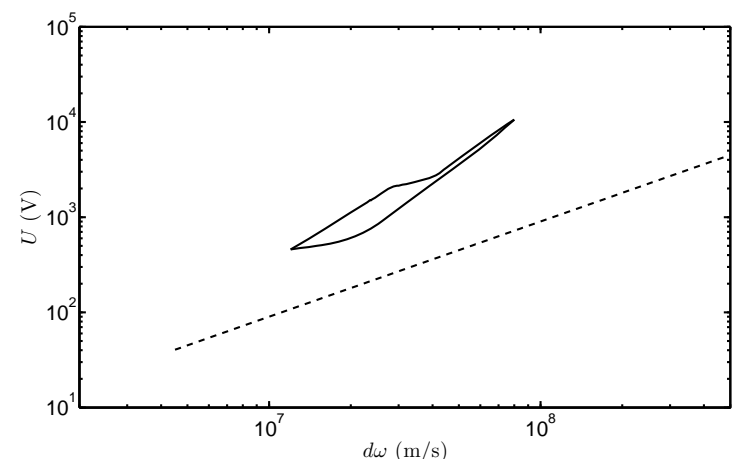

Figure 4: The appearance of the classic Hatch-Williams diagram for a pulse length of 7 field periods. The physical parameters are the same as in Fig. 1.

\section{The 20 gap threshold}

The application of the 20 gap crossing rule is described in [3], and it is appropriate to compare our results with those of the 20 gap rule. The proceedure for doing this approximately is rather straightforward. Electrons cross the gap in the time $\tau_{n}=n T / 2$. The number of times an electron bunch belonging to a certain mode will cross the gap in the pulse time is thus $m=\left[\tau_{p} / \tau_{n}\right] \approx 2 \tau_{p} /(n T)$. Only modes that have $m \geq 20$ are assumed to be able to lead to multipactor. Hence

$$
n \leq \frac{1}{10} \frac{\tau_{p}}{T}
$$

For $\tau_{p} / T<10$, multipactor is impossible. The first $(n=1)$ resonance zone becomes "activated" when $\tau_{p} / T=10$, the second $(n=3)$ when $\tau_{p} / T=30, n=$ 5 when $\tau_{p} / T=50$ and so on. To find the lower breakdown voltage corresponding to each resonance zone, we consider the time it takes to cross the gap. In a resonant discharge, close to the lower breakdown threshold, the electrons cross the gap in roughly the time $d /\left(v_{\omega}+v_{e}\right)$. Putting this equal to $\tau_{n}$ we find

$$
v_{\omega} \approx \frac{d}{\tau_{n}}-v_{e}
$$

Which gives us the breakdown voltage from

$$
U=\frac{m \omega d v_{\omega}}{e}
$$

However, we also require $v_{1} \leq 2 v_{\omega}+v_{e} \leq v_{2}$, which means

$$
\frac{2 d \omega}{\pi\left(v_{e}+v_{2}\right)} \leq n \leq \frac{2 d \omega}{\pi\left(v_{e}+v_{1}\right)}
$$

This gives us a bound on the resonance zones that may appear in the HatchWilliams diagram. How many of these zones that will actually appear depends 
on the pulse length according to Eq. (13). The application of formulas (13), (15), and (16) yields different values for the breakdown voltage as a function of pulse length over field period. In each interval belonging to a given resonance order, the breakdown voltage is a certain constant. Using the full resonance theory presented in section 3 yields a similar set of thresholds. However, these are not exactly constant in each interval, but can vary quite a lot, depending on the resonance order. Comparison between the twenty gap rule results and the results of the full resonant theory are seen in Figs. 5- 8, which will be discussed in the next section.

\section{$5 \quad$ Breakdown voltage and pulse length}

The Hatch-William diagrams in section 2 represent an idealized picture of the multipactor phenomenon in pulsed signals. The detailed result crucially depends on the combination of material parameters and the gap-frequency product. However, some qualitative features could be emphasized. For large values of the product $d \cdot \omega$, only higher order multipactor modes can be important (since the resonance zones of lower order involve very high impact velocities and SEY values below unity). These zones are very narrow and disappear when the pulse becomes too short. Consequently, one would expect a ladder-like profile for the breakdown voltage, where the voltage jumps between the different resonance zones, becoming successively higher as the pulse becomes shorter. On the other hand, for very small values of $d \cdot \omega$, only the first resonance zone will be important. This zone is quite wide and the effective SEY value varies significantly over the width of the zone. This will lead to a smoothly increasing breakdown voltage for decreasing pulse lengths. In Figs. $5-8$, the form of the breakdown curves are shown for four different values of $d \cdot \omega$. The maximum pulse length was chosen to be $\tau_{p} / T=100$ and only discrete values of $\tau_{p} / T$ were used. When using classical resonance theory (i.e. neglecting emission velocity spread), it becomes quite cumbersome to include results for very long pulses for large values of $d \cdot \omega$, since it would involve many higher order resonance zones. On the other hand, it is easy to estimate the highest order of resonance that will be relevant for a given pulse length. From Eq. (10) it can be inferred that

$$
n_{\max }=\frac{\tau_{p}}{3 T} \frac{\ln \sigma_{\max }}{\ln 10}
$$

For $\max \left(\tau_{p} / T\right)=100$ this yields $n_{\max }=15$ as the maximum resonance order that can be important, regardless of the gap width.

There are few experimental results available in the published literature concerning multipactor breakdown in pulsed signals that allow a detailed comparison. However, in Ref. [6], the multipactor breakdown power was measured as a function of pulse length, making possible at least a qualitative comparison with the theoretical results obtained in the present work. In the experiment, the breakdown power was found to be almost constant as the pulse width decreased from $100 \mu \mathrm{s}$ down to approximately $5 \mu$ s and then the characteristic 
pulse hardening was observed for further decreasing pulse width c.f. Fig.5. It is also interesting to note that in the experiment there is a jump down in breakdown threshold as the pulse length is increased from $100 \mu \mathrm{s}$ to $200 \mu \mathrm{s}$. This feature was commented upon in Ref. [6] as "unclear" , but was confirmed by repeated measurements. Clearly this jump is in good qualitative agreement with the present findings and can be explained as a transition to another resonance order.

In Figs. $5-8$ one can see the breakdown threshold as a function of pulse length for $d \cdot \omega=10^{7}, 3 \times 10^{7}, 10^{8}$, and $2 \times 10^{8}(\mathrm{~m} / \mathrm{s})$ respectively. In Fig. 5 we have $d \cdot \omega=10^{7}(\mathrm{~m} / \mathrm{s})$, and only the first resonance zone is relevant. Since the first resonance zone is quite wide, the SEY value varies significantly over the width of the resonance region and the breakdown curve has a distinctive smooth appearance, steadily increasing with decreasing pulse length. This is the so-called pulse hardening effect. Fig. 6 shows the case of $d \cdot \omega=3 \times 10^{7}$ $(\mathrm{m} / \mathrm{s})$. Inspecting Fig. 1 one can infer that the two first resonance zones are responsible for the discharge. The jump between the two resonance zones is very clear and the sudden increase in breakdown voltage when going from $n=3$ to $n=1$ is almost twice the value for $n=3$. This agrees qualitatively with the results found in Ref. [6]. In Fig. 7 we have $d \cdot \omega=10^{8}(\mathrm{~m} / \mathrm{s})$, and we can see the resonance zones corresponding to $n=3,5$ and 7 . The jumps between the resonance zones are still quite clear. Finally, in Fig. 8 we have $d \cdot \omega=2 \times 10^{8}$ $(\mathrm{m} / \mathrm{s})$, and we can see the thresholds corresponding to $n=5,7,9$, and 11 . The jumps between the resonance zones are less distinctive in comparison to Fig. 6 and 7 .

An inspection of figures $5-8$ reveals that for large values of $d \cdot \omega$, the twenty gap rule and the more sophisticated calculations give qualitatively the same result. The actual values for the breakdown thresholds, and the point of inception of a new resonance zone do not agree completely, and e.g. in figure 8 the twenty gap crossing rule states that electrons belonging to $n=11$ are of no consequence. This does not agree with the more detailed calculations. Furthermore, for low values of $d \cdot \omega$, the thresholds show a certain dependence on $\tau_{p} / T$ within the resonance intervals (see figs. 5 and 6 ). This feature is not predicted by the twenty gap crossing rule.

Throughout this paper we assumed a multiplication factor $\exp (m \ln \sigma) \approx$ $10^{6}$. This number is based on a very rough estimate of the average SEY and using the 20-gap rule. It is therefore of interest to investigate the results of changing this factor. In Fig. 9 the breakdown threshold is shown for the case of first order resonance for three different values of the multiplication factor: $10^{4}, 10^{6}$, and $10^{8}$. Clearly, the qualitative results are identical, and the three curves all show the same continuous increase in the threshold field with decreasing pulse length. However, by decreasing the multiplication factor we move the start of this increase to lower pulse lengths, and similarily, by increasing the multiplication factor we move the start to longer pulses. This is perfectly natural, since a lower number of required electron number increase in a fixed number of gap crossings require a lower SEY and consequently a lower voltage. Whereas higher electron number increase requires higher voltages. Looking at Fig. 10 


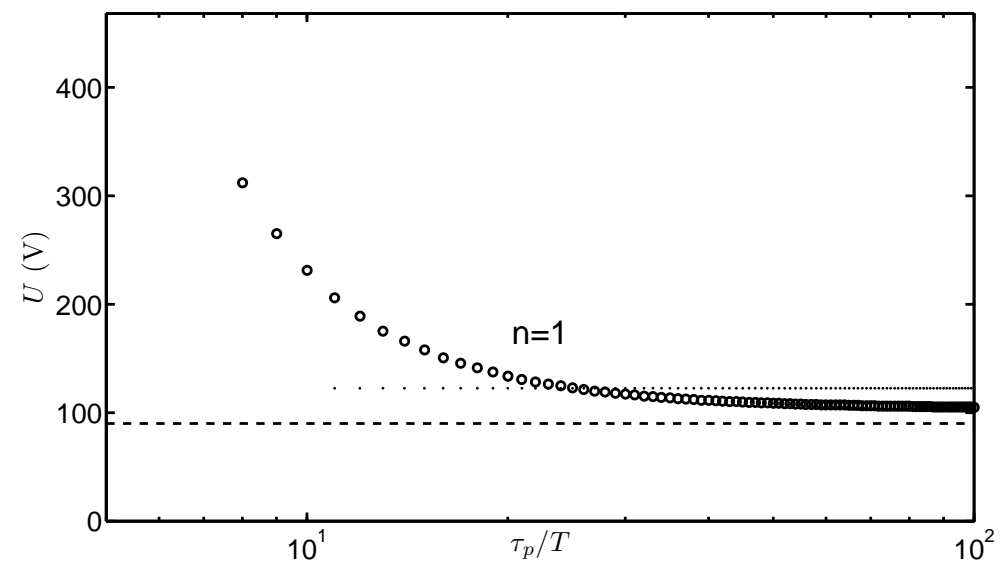

Figure 5: The circles represent the lower breakdown voltage as a function of $\tau_{p} / T$ for $d \cdot \omega=10^{7} \mathrm{~m} / \mathrm{s}$. The material properties are the same as in Fig. 1. The dots represent the lower threshold as given by the twenty gap crossing rule (see section 4). The dashed straight line represents the breakdown voltage predicted by Eq. (11).

we see the same situation but for a gap where 1'st and 3'rd order multipactor is relevant. The effect of changing the multiplication factor is simply to shift the transition between the two resonances either to lower pulse lengths for a lower multiplication factor, or higher pulse lengths for a higher multiplication factor.

It should be noted that complicated rf signals inside communication lines does not always lead to an increase in the multipactor threshold. Specifically a recent study [15] revealed that inside a relatively wide gap, a decrease in the multipactor threshold is possible in the case of digitally modulated signals. Such a decrease can be attributed to a very general phenomenon known as stochastic electron heating in microwave fields [16]. In fact, when the rf electric field switches phase in a stochastic manner, electrons can acquire more energy than in the case of a pure monochromatic signal with the same amplitude, provided that the electron transit times are long enough, and that the phase jumps do not occur with too high frequency. Therefore particular attention should be paid to multipactor predictions inside wide gaps in the case of complex rf signals. 


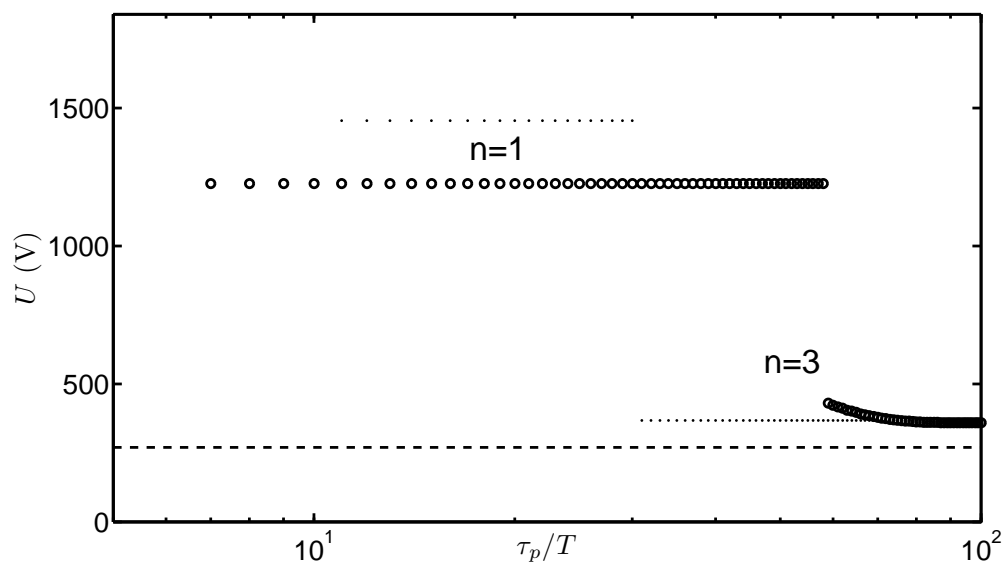

Figure 6: The lower breakdown voltage as a function of $\tau_{p} / T$ for $d \cdot \omega=3 \times 10^{7}$ $\mathrm{m} / \mathrm{s}$.

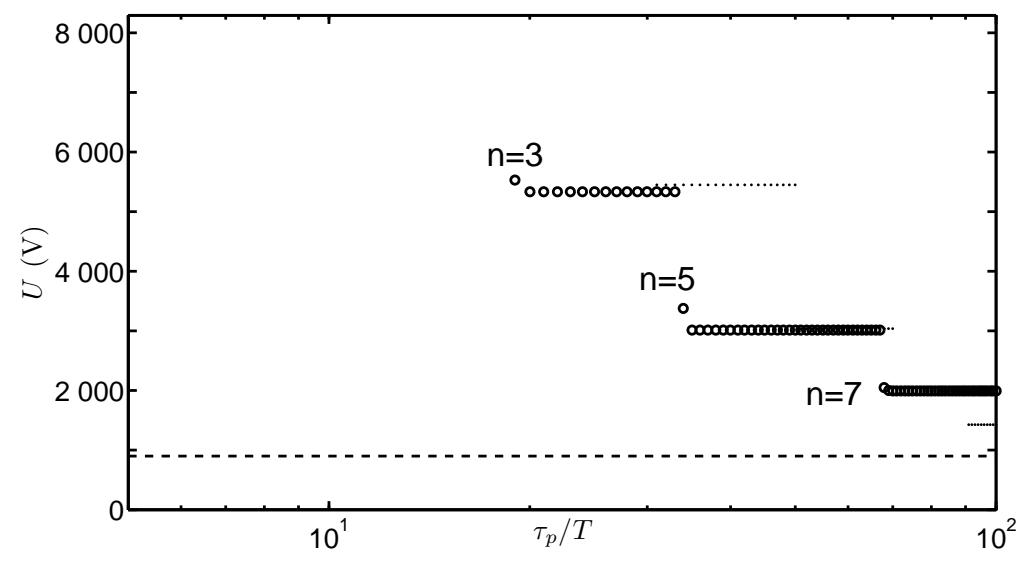

Figure 7: The lower breakdown voltage as a function of $\tau_{p} / T$ for $d \cdot \omega=10^{8}$ $\mathrm{m} / \mathrm{s}$. 


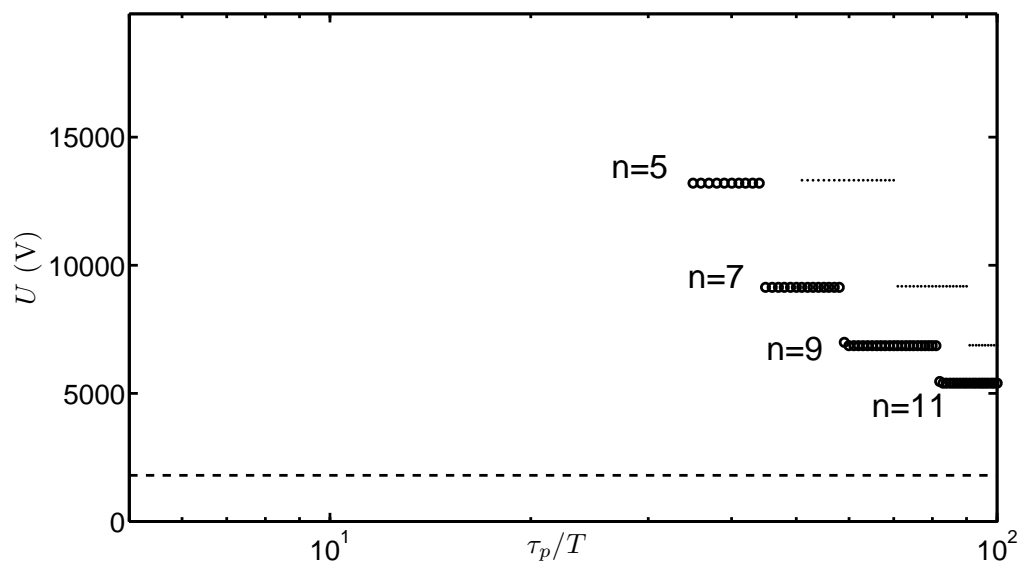

Figure 8: The lower breakdown voltage as a function of $\tau_{p} / T$ for $d \cdot \omega=2 \times 10^{8}$ $\mathrm{m} / \mathrm{s}$.

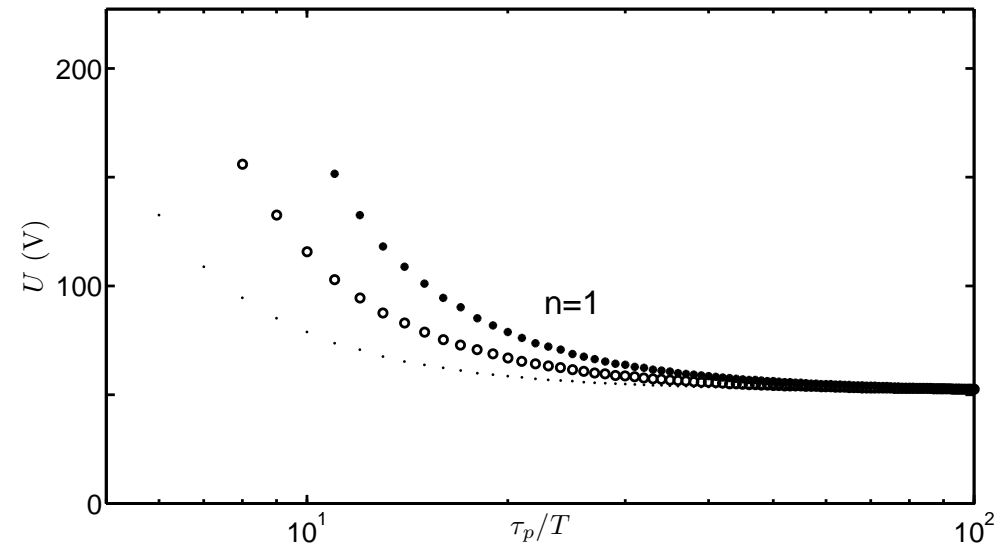

Figure 9: The lower breakdown voltage as a function of $\tau_{p} / T$ for $d \cdot \omega=10^{7} \mathrm{~m} / \mathrm{s}$, and three different values of the multiplication factor: $N / N_{0}=10^{4}$ (marked by small dots), $10^{6}$ (circles), and $10^{8}$ (big dots). 


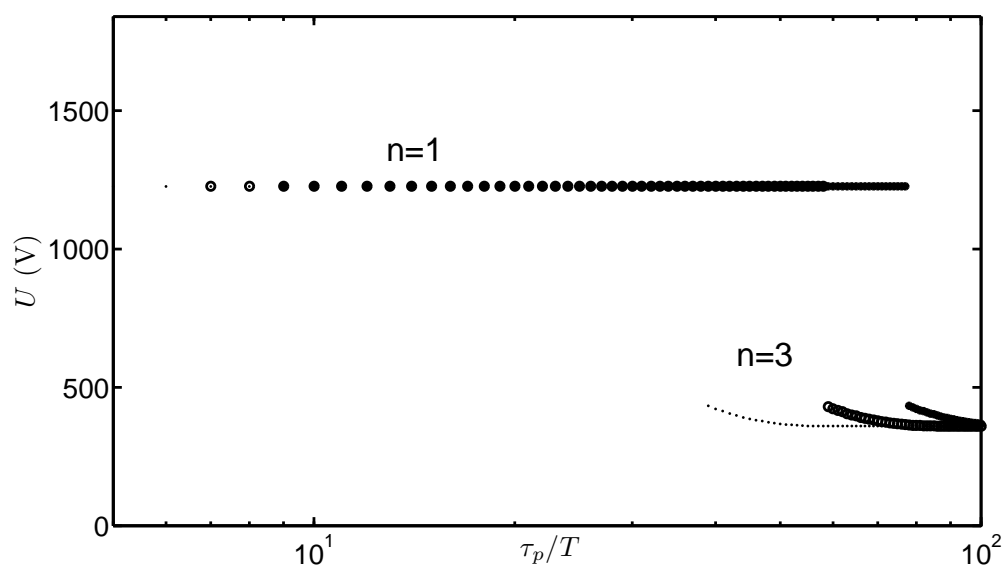

Figure 10: The lower breakdown voltage as a function of $\tau_{p} / T$ for $d \cdot \omega=$ $3 \times 10^{7} \mathrm{~m} / \mathrm{s}$, and three different values of the multiplication factor: $N / N_{0}=10^{4}$ (marked by small dots), $10^{6}$ (circles), and $10^{8}$ (big dots).

\section{Conclusions}

A first theoretical investigation has been made of the effect of finite pulse length on the multipactor breakdown condition in microwave signals, within the classical resonant theory for parallel plates. It is found that decreasing pulse length tends to successively eliminate higher order resonance zones in a step like fashion and to lead to a "pulse hardening" of lower order modes where the breakdown field of the zones smoothly increases as the pulse become shorter, eventually to completely eliminate the multipactor effect for sufficiently short pulses. These features are in good qualitative agreement with recently published experimental results. We also found that the qualitative features of the different breakdown curves had no dependence on the choice of the multiplication factor, but that the actual values for the pulse length where the pulse hardening and jumping between resonance orders are shifted. Since the multiplication factor depends so heavily on the number of background electrons, it might be able to verify this effect experimentally using different seeding sources. In the space community, the twenty gap crossing rule is used to judge whether a system is susceptible to multipactor. However, it was shown above that only the basic features of the breakdown threshold can be replicated using the twenty gap rule. The pulse hardening effect is missed completely, and the breakdown threshold is generally quite different. Aside from shedding light on some recent experimental results, and providing a first theoretical investigation of the resonant multipactor process in pulsed signals, the study also gives practical guidelines for designing microwave signals. In particular, formulas are given for estimating how short a pulse needs to be to completely eliminate resonant multipactor, and how to 
estimate the resonance order and breakdown voltage in the case of pulsed resonant multipactor in a parallel plate system. We did not take into consideration the possibility of electron build-up over several pulses. This can be the case when the pulses are sufficiently long, and the time between pulses is sufficiently short for the electron number not to decay completely. In systems with very large dimensions there is also the possibility of an electron avalanche even if the pulses are very short and well separated, for electrons will be accelerated during the pulse, and then drift across the gap, to eventually collide with a wall. If the time of the collision is synchronous with another pulse, these impacts can lead to secondary electrons with the right speed to repeat the process, and eventually lead to a multipactor breakdown.

\section{References}

[1] S. Anza, C. Vicente, B. Gimeno, V. E. Boria, and J. Armendáriz, "Longterm multipactor discharge in multicarrier systems", Phys. Plasmas, 14, $082112(2007)$.

[2] A. J. Marrison, R. May, J. D. Sanders, A. D. Dyne, A. D. Rawlins, and J. Petit, A Study of Multipaction in Multicarrier RF Components, AEA Technology for ESTEC, AEA Ref. No. AEA/TYKB/31761/01/RP/05 Issue 1, (Culham, UK, 1997).

[3] Space Engineering, Multipactor Design, and Test, ECSS-E-20-01-01A, May 5 (2003).

[4] S. Anza, M. Mattes, J. Armendariz, J. Gil, C. Vicente, B. Gimeno, V. E. Boria, and D. Raboso, "RF Breakdown Prediction for Microwave Passive Components in Multi-carrier Operation", pages 375-381 in Ultra-Wideband, Short Pulse Electromagnetics 9, Springer, 2010.

[5] S. Anza, M. Mattes, C. Vicente, J. Gil, D. Raboso, V. E. Boria, and B. Gimeno, "Multipactor theory for multicarrier signals", Phys. Plasmas, 18, 032105 (2011).

[6] N. Le Gallou, C. Miquel-Espanya, S. Matinfar, C. Ernst and F. De Paolis, "Multipactor thresholds and margins using realistic modulated signals", MULCOPIM 2011 Valencia, Spain 21-23 Sept. 2011

[7] S. Anza, C. Vicente, J. Gil, M. Mattes, D. Wolk, U. Wochner, V. E. Boria, B. Gimeno, and D. Raboso, "Prediction of Multipactor Breakdown for Multicarrier Applications: The Quasi-Stationary Method", IEEE Trans. Microw Theory Tech., 60, 7, 2093 (2012).

[8] V. E. Semenov, M. Buyanova, D. Anderson, M. Lisak, R. Udiljak, and J. Puech, "Multipactor in Microwave Transmission Systems Using Quadrature Phase Shift Keying", IEEE Trans. Plasma Sci., 38, 4, 915 (2010). 
[9] A. D. MacDonald, Microwave Breakdown in Gases, John Wiley and Sons, New York (1966)

[10] A. J. Hatch, and H. B. Williams, "Multipacting Modes of High-Frequency Gaseous Breakdown", Phys. Rev., 112, 3, 681 (1958).

[11] J. Rasch, and J. F. Johansson, "Non-Resonant Multipactor - A Statistical Model", Phys. Plasmas, 19, 12, 123505 (2012).

[12] A. Kryachev, M. Buyanova, V. Semenov, D. Anderson, M. Lisak, J. Puech, L. Lapierre and J. Sombrin, "Hybrid resonant modes of two-sided multipactor and transition to the polyphase regime", Physics of Plasmas, 9, $4736(2002)$

[13] V. E. Semenov, E. Rakova, D. Anderson, M. Lisak, and J. Puech, "Multipactor in Rectangular Waveguides", Phys. Plasmas, 14, 3, 033501 (2007).

[14] J. R. M. Vaughan, "A New Formula for Secondary Emission Yield", IEEE Trans. Electron Dev., 36, 9, 1963 (1989).

[15] D. González-Iglesias, O. M. Belda, O. Monerris, B. Gimeno, V. E. Boria, D. Raboso, V. E. Semenov, "Analysis of Multipactor Effect Using a Phase-Shift Keying Single-Carrier Digital Modulated Signal", IEEE Trans. Electron Dev., 60, 8, 2664 (2013).

[16] S. Puri, "Stochastic heating of plasma electrons using microwave noise", Plasma Phys., 16, 517 (1974). 